\section{Tendências da hanseníase no Tocantins, um estado hiperendêmico do Norte do Brasil, 2001-2012}

\author{
Leprosy trends in Tocantins, a hyperendemic State \\ in the North of Brazil, 2001-2012
}

\section{Tendencias de la lepra en Tocantins, un estado hiperendémico del norte de Brasil, 2001-2012}

\author{
1 Secretaria de Estado da \\ Saúde do Tocantins, Palmas, \\ Brasil. \\ 2 Universidade Federal do \\ Ceará, Fortaleza, Brasil. \\ 3 Instituto Federal de \\ Educação, Ciência e \\ Tecnologia do Ceará, \\ Caucaia, Brasil. \\ 4 School of Public Health \\ Tropical Medicine and \\ Rehabilitation Sciences, \\ James Cook Universit \\ Townsville, Australia. \\ Correspondência \\ L. D. Monteiro \\ Secretaria de Estado da \\ Saúde do Tocantins. \\ Praça dos Girassois s/n, Plano \\ Diretor Sul, Palmas, TO \\ 77015-007, Brasil. \\ lorenamonteiro3@hotmail.com
}

\begin{abstract}
Leprosy is hyperendemic in the State of Tocantins, Brazil. The aim of the study was to analyze trends in leprosy indicators in Tocantins from 2001 to 2012, based on analysis of data from the Information System for Notifiable Diseases (SINAN), including incident cases living in Tocantins. The study calculated leprosy indicators and analyzed time trends using polynomial regression. There was a significant downward trend in overall case detection $\left(R^{2}=0.40\right.$; $p<0.05)$ and proportion of paucibacillary cases $\left(R^{2}=0.81\right)$. Detection rates remained stable in individuals $<15$ years $\left(R^{2}=0.48 ; p>0.05\right)$ and cases with grade 2 physical disability $\left(R^{2}=0.37\right.$; $p>0.05)$, as did the proportion of grade 2 cases $\left(R^{2}=0.49 ; p>0.05\right)$. There were significant increases in the proportions of cases with grade 1 disability $\left(R^{2}=0.82 ; p<0.05\right)$ and multibacillary cases $\left(R^{2}=0.81 ; p<0.05\right)$. Tocantins has regions with high transmission and late diagnosis of leprosy, indicating heterogeneous dissemination of the disease in the time trend analysis.
\end{abstract}

Leprosy; Neglected Diseases; Endemic Diseases
Lorena Dias Monteiro 1,2

Francisco Rogerlândio Martins-Melo 2,3 Aline Lima Brito 2

Mauricélia da Silveira Lima 2 Carlos Henrique Alencar 2

Jorg Heukelbach 2,4

\section{Resumo}

A hanseníase é hiperendêmica no Estado do Tocantins, Brasil. O objetivo do estudo foi analisar as tendências dos indicadores da hanseníase no Tocantins em 2001-2012. Análise de dados advindos do Sistema de Informação de Agravos de Notificação (SINAN). Incluíram-se casos novos de residentes no Tocantins. Calcularam-se os indicadores da hanseníase e analisaram-se as tendências temporais por meio de regressão polinomial. Houve tendência significativa e decrescente para a detecção geral $\left(R^{2}=0,40 ; p<0,05\right)$ e proporção de casos paucibacilares $\left(R^{2}=0,81\right)$. Foi estável a detecção em $<15$ anos $\left(R^{2}=0,48\right.$; $p>0,05)$, detecção de casos com grau 2 de incapacidade física $\left(R^{2}=0,37 ; p>0,05\right)$ e proporção de casos com grau $2\left(R^{2}=0,49 ; p>0,05\right)$. Houve aumento significativo para a proporção de casos com grau 1 de incapacidade $\left(R^{2}=0,82 ; p<0,05\right)$ e proporção de casos multibacilares $\left(R^{2}=0,81\right.$; $p<0,05)$. O Tocantins apresenta regiões com alta transmissão e diagnóstico tardio da hanseníase, apontando a expansão da doença de forma heterogênea na análise temporal.

Hanseníase; Doenças Negligenciadas; Doenças Endêmicas 


\section{Introdução}

A hanseníase, doença causada por Mycobacterium leprae, mantém-se como um processo infeccioso crônico de elevada magnitude em vários países do mundo 1. Em 2012, foram registrados aproximadamente 233 mil casos novos da doença mundialmente, sendo que 16 países registraram acima de 1.000 casos 1 . O continente americano registra aproximadamente $17 \%$ de todos esses casos, com o Brasil responsável por $93 \%$ dos casos 1 . Embora o Brasil registre decréscimos nos coeficientes de detecção de casos novos de hanseníase, em 2012 foram registrados 33.303 casos, sendo as regiões Norte, Nordeste e Centro-oeste as mais endêmicas, com áreas de importante manutenção da transmissão 2,3 .

A meta de eliminação da hanseníase como problema de saúde pública, com menos de um caso por 10 mil habitantes, foi definida pela Organização Mundial da Saúde (OMS) e seguida pelo Ministério da Saúde. Porém, esse alvo tem sido um desafio a ser alcançado em algumas regiões em consequência da sua complexidade 1,4,5,6.

No Estado do Tocantins, a hanseníase é considerada hiperendêmica, de acordo com os parâmetros do Ministério da Saúde. Em 2012, o estado ocupou o segundo lugar no ranking brasileiro, com um coeficiente de detecção de casos novos de hanseníase de 73,4 por 100 mil habitantes. O coeficiente de detecção em menores de 15 anos de idade foi de 22,4 por 100 mil habitantes, sendo o primeiro no país (Coordenação Geral de Hanseníase e Doenças em Eliminação, Secretaria de Vigilância em Saúde, Ministério da Saúde. Banco de dados concedido por solicitação no Portal da Transparência. http://www.portal.saude.gov.br).

Dada a complexidade epidemiológica da hanseníase no Estado do Tocantins 7,8,9, a escassez de estudos sistemáticos sobre o seu comportamento e distribuição, bem como a necessidade de melhores subsídios para intensificar medidas de controle em áreas hiperendêmicas, este estudo objetivou caracterizar os padrões epidemiológicos e tendências temporais dos indicadores da hanseníase no Estado do Tocantins, no período de 2001 a 2012.

\section{Material e métodos}

\section{Área de estudo}

O Estado do Tocantins, localizado na Região Norte do Brasil, tem extensão territorial de $277.622 \mathrm{~km}^{2}$, e é dividido em 139 municípios. A população estimada foi de 1,4 milhão de habitantes em 2013, com crescimento de $18,4 \%$ des- de o ano 2000 (Instituto Brasileiro de Geografia e Estatística. http://www.ibge.gov.br/estadosat/ perfil.php?sigla=to, acessado em 12/Mar/2014). O estado está dividido em oito regiões de saúde formadas de acordo com os municípios, população e densidade demográfica para oferta de ações e serviços mínimos em cada território. A região de saúde de Augustinópolis é formada por 24 municípios e está localizada no extremo Norte; a região de Araguaína agrega 17 municípios e está ao Norte; a região de Guaraí, com 23 municípios, está no Centro-norte; a região de Paraíso, 16 municípios, situada no Centro-oeste; a região de Palmas é formada por 14 municípios e está no Centro-leste; a região de Porto Nacional, 12 municípios, está ao Leste; região de Gurupi, 18 municípios, é localizada no Sudoeste; e a região de Dianópolis com 15 municípios, no Sudeste do estado.

\section{Desenho e população de estudo}

Trata-se de um estudo baseado em dados secundários, oriundos do Sistema de Informação de Agravos de Notificação (SINAN). Foram incluídos todos os casos novos de hanseníase de residentes no Estado do Tocantins no período de 2001 a 2012 .

\section{Fonte de dados}

Os dados foram obtidos no SINAN, provenientes das fichas de notificações compulsórias, que consistem em um formulário padronizado com informações sociodemográficas e clínicas preenchidas por profissionais de saúde. O banco de dados com todas as notificações nacionais foi obtido junto à Coordenação Geral de Hanseníase e Doenças em Eliminação do Ministério da Saúde (CGHDE-MS). Foram excluídos os registros com erro de diagnóstico, duplicidades e com códigos incompatíveis dos municípios do estado. Foram também excluídos casos de residentes em outros estados.

Os dados populacionais foram obtidos no Instituto Brasileiro de Geografia e Estatística (IBGE), com base em dados dos censos da população do estado (2010) e estimativas populacionais para os anos intercensitários (2001 a 2012) (Instituto Brasileiro de Geografia e Estatística. http://www.ibge.gov.br/estadosat/perfil. php?sigla=to, acessado em 12/Mar/2014).

\section{Análise dos dados}

Para análise descritiva, foram selecionadas as variáveis segundo registros de casos por ano e região de saúde. Calcularam-se os indicadores 
preconizados pelo Programa Nacional para Avaliação e Monitoramento da Hanseníase: coeficiente de detecção de casos novos, por $100 \mathrm{mil}$ habitantes, na população geral (indica a magnitude da hanseníase em uma área); coeficiente de detecção em menores de 15 anos de idade, por 100 mil habitantes (indica a transmissão ativa da doença); proporção de casos com formas clínicas avançadas (indica diagnóstico tardio); proporção de casos paucibacilares (indica diagnóstico precoce) e número de casos novos com grau $2 \mathrm{de}$ incapacidade detectada em uma população (indica subdetecção); e proporção de casos novos com grau 2 de incapacidade entre todos os casos novos detectados durante o ano, que é um indicador usado para avaliar a qualidade das atividades do programa de controle 10. Os indicadores foram calculados utilizando-se como unidade geográfica de análise o Estado do Tocantins e as oito regiões de saúde.

Para a análise da tendência da hanseníase foram utilizados modelos de regressão polinomial para as séries temporais. A equação de regressão aplicada foi a que melhor descreveu a relação existente entre a variável dependente $\mathrm{Y}$ (indicadores de monitoramento e avaliação para hanseníase) e a variável independente X (ano de estudo). Para evitar autocorrelação entre os termos da equação da regressão, foi utilizado o artifício de centralizar a variável ano, transformando-a em ano calendário, com ponto médio da série os anos de 2006 e 2007 11. O primeiro modelo testado foi o de regressão linear simples $(\log \mathrm{Y}=\beta 0$ $+\beta 1 \mathrm{X}$ ) e, posteriormente, os modelos de ordem maior: segundo grau ou parabólico $(\log \mathrm{Y}=\beta 0+$ $\left.\beta 1 \mathrm{X}+\beta 2 \mathrm{X}^{2}\right)$ e terceiro grau $(\log \mathrm{Y}=\beta 0+\beta 1 \mathrm{X}+\beta 2$ $\left.X^{2}+\beta 3 X^{3}\right) 11$. As tendências foram consideradas estatisticamente significativas quando os modelos apresentaram valor de $\mathrm{p}<0,05$. Foi considerado como melhor modelo aquele que apresentou maior coeficiente de determinação ( $\left.\mathrm{R}^{2}\right)$. Quando dois modelos foram semelhantes estatisticamente para a mesma localidade, optou-se pelo modelo mais simples, ou seja, de menor ordem.

Os dados foram analisados utilizando-se o sotware Stata, versão 11.2 (Stata Corp., College Station, Estados Unidos).

\section{Aspectos éticos}

Este estudo foi aprovado pelo Comitê de Ética em Pesquisa (CEP) da Universidade Federal do Ceará (parecer no 544.962 de 28 de fevereiro de 2014).

\section{Resultados}

No período de 2001 a 2012, foi registrado um total de 14.532 casos novos de hanseníase em residentes no Estado do Tocantins. Houve maior registro de casos em 2006 ( $\mathrm{n}=1.450 ; 10 \%$ ). A região de saúde de Gurupi apresentou a maior ocorrência de casos (2.952; 20,3\%). A frequência de casos nas demais regiões foi: Palmas (2.886; 19,9\%), Araguaína (2.441; 16,8\%), Guaraí (2.179; 15\%), Augustinópolis (1.310; 9\%), Paraíso (1.116; $7,7 \%)$, Porto Nacional (796; 5,5\%); Dianópolis (536; 3,7\%). Um total de 316 (2,1\%) casos não especificava a região de residência.

Na Tabela 1, os resultados apontam tendência de declínio significativo para o coeficiente de detecção geral (por 100 mil habitantes) (R2: 40\%; $\mathrm{p}=0,025)$ e proporção de casos paucibacilares (R2: 81\%; $\mathrm{p}<0,001)$.

Uma estabilidade foi observada para o coeficiente de detecção em menores de 15 anos de idade (por 100 mil habitantes) $\left(\mathrm{R}^{2}: 48 \% ; \mathrm{p}=0,050\right)$ (Figura 1), coeficiente de detecção de casos com grau 2 de incapacidade física (por 100 mil habitantes) (R2: $37 \% ; \mathrm{p}=0,377)$ e proporção de casos com grau 2 de incapacidade física entre os avaliados (R2: 49\%; $\mathrm{p}=0$,499) (Tabela 1; Figura 2).

Um crescimento foi encontrado para a proporção de casos com grau 1 de incapacidade física no diagnóstico (R2: 82\%; p < 0,001) (Figura 2) e proporção de casos multibacilares (R2: $81 \%$; $\mathrm{p} \leq$ 0,001) (Tabela 1; Figuras 2 e 3).

Apesar da redução significativa do coeficiente de detecção geral da doença no estado, este padrão foi heterogêneo nas regiões de saúde. Esse indicador ficou estável nas regiões de Araguaína $(\mathrm{p}=0,075)$ no Norte do estado, Augustinópolis ( $\mathrm{p}$ $=0,125$ ) ao extremo Norte, bem como a região de Dianópolis ( $\mathrm{p}=0,133$ ) no Sudeste do Tocantins. As demais regiões, localizadas no Centro-sul e Sudoeste do estado, apresentaram declínio significativo na detecção de novos casos $(\mathrm{p}<0,005)$ (Tabela 1).

A detecção de casos novos em menores de 15 anos de idade ao longo da série histórica apresentou declínio significativo para as regiões de Augustinópolis $\left(\mathrm{R}^{2}=64 \% ; \mathrm{p}=0,010\right)$ no extremo Norte, Guaraí ( $\mathrm{R}^{2}=35 \%$; $\mathrm{p}=0,040$ ) localizada no centro do estado, e para as regiões de Paraíso $\left(\mathrm{R}^{2}=\right.$ $69 \%$; $\leq 0,001)$ no Centro-oeste. Nas outras cinco regiões de saúde houve estabilidade $(\mathrm{p}>0,05)$ (Tabela 1).

Os coeficientes de detecção de casos novos de hanseníase com grau 2 de incapacidade física apresentaram estabilidade em todas as regiões de saúde ( $\mathrm{p}>0,05)$ na série avaliada, explicados pelo modelo de terceiro grau da regressão polinomial (Tabela 1). 
Tabela 1

Tendência dos indicadores de hanseníase no Estado do Tocantins e suas regiões de saúde (por 100 mil habitantes), Brasil, 2001-2012.

\begin{tabular}{|c|c|c|c|c|}
\hline Indicadores & Modelo * & $\mathbf{R}^{2}$ ** & Valor de p & Tendência \\
\hline Coeficiente de detecção geral & $y=93,329-2,234 x$ & 0,40 & 0,025 & Decrescente \\
\hline Coeficiente de detecção em menores de 15 anos & $y=26,889-0,143 x-0,207 x^{2}$ & 0,48 & 0,050 & Estabilidade \\
\hline Coeficiente de detecção com grau 2 de incapacidade física & $y=4,741-1,57 x-0,43 x^{2}+0,09 x^{3}$ & 0,30 & 0,377 & Estabilidade \\
\hline Proporção de casos com grau 1 de incapacidade física & $y=20,094+1,340 x$ & 0,82 & 0,000 & Crescente \\
\hline Proporção de casos com grau 2 de incapacidade física & $y=5,593-0,088 x-0,22 x^{2}+0,11 x^{3}$ & 0,24 & 0,499 & Estabilidade \\
\hline Proporção de casos multibacilares & $y=45,576+1,523 x$ & 0,81 & 0,000 & Crescente \\
\hline Proporção de casos paucibacilares & $y=54,396-1,513 x$ & 0,81 & 0,000 & Decrescente \\
\hline \multicolumn{5}{|l|}{ Regiões de saúde (detecção geral por 100 mil habitantes) } \\
\hline Augustinópolis & $y=68,355+2,761 x-0,739 x^{2}-0,070 x^{3}$ & 0,49 & 0,125 & Estabilidade \\
\hline Araguaína & $y=94,125-2,078 x-0,647 x^{2}-0,146 x^{3}$ & 0,55 & 0,075 & Estabilidade \\
\hline Guaraí & $y=158,151-9,570 x-2,128 x^{2}-0,280 x^{3}$ & 0,69 & 0,019 & Decrescente \\
\hline Palmas & $y=96,997-4,990 x$ & 0,60 & 0,003 & Decrescente \\
\hline Porto Nacional & $y=78,663+1,969 x-0,911 x^{2}$ & 0,60 & 0,016 & Decrescente \\
\hline Paraíso & $y=87,127-4,681 x$ & 0,61 & 0,003 & Decrescente \\
\hline Gurupi & $y=147,691-5,438 x$ & 0,47 & 0,014 & Decrescente \\
\hline Dianópolis & $y=57,790+2,982 x-0,713 x^{2}-0,73 x^{3}$ & 0,48 & 0,133 & Estabilidade \\
\hline \multicolumn{5}{|l|}{ Detecção em < 15 anos de idade } \\
\hline Augustinópolis & $y=23,014+1,274 x-0,457 x^{2}$ & 0,642 & 0,010 & Decrescente \\
\hline Araguaína & $y=31,077+0,659 x-0,320 x^{2}$ & 0,363 & 0,131 & Estabilidade \\
\hline Guaraí & $y=14,124-0,704 x$ & 0,356 & 0,040 & Decrescente \\
\hline Palmas & $y=71,666+1,520 x-0,278 x^{2}-0,129 x^{3}$ & 0,329 & 0,388 & Estabilidade \\
\hline Porto Nacional & $y=19,437+2,828 x-0,284 x^{2}-0,104 x^{3}$ & 0,533 & 0,092 & Estabilidade \\
\hline Paraíso & $y=25,282-2,456 x$ & 0,693 & 0,001 & Decrescente \\
\hline Gurupi & $y=38,838-1,637 x+0,279 x^{2}+0,33 x^{3}$ & 0,164 & 0,679 & Estabilidade \\
\hline Dianópolis & $y=12,168+1,465 x+0,086 x^{2}-0,077 x^{3}$ & 0,168 & 0,668 & Estabilidade \\
\hline \multicolumn{5}{|l|}{ Detecção de casos com grau 2} \\
\hline Augustinópolis & $y=3,597-0,0385 x-0,073 x^{2}+0,017 x^{3}$ & 0,245 & 0,497 & Estabilidade \\
\hline Araguaína & $y=3,240+0,999 x+0,003 x^{2}-0,002 x^{3}$ & 0,025 & 0,976 & Estabilidade \\
\hline Guaraí & $y=4,426-0,335 x-0,004 x^{2}+0,031 x^{3}$ & 0,382 & 0,254 & Estabilidade \\
\hline Palmas & $y=4,101-0,435 x-0,016 x^{2}+0,015 x^{3}$ & 0,152 & 0,706 & Estabilidade \\
\hline Porto Nacional & $y=5,492+0,033 x-0,103 x^{2}+0,09 x^{3}$ & 0,532 & 0,229 & Estabilidade \\
\hline Paraíso & $y=4,113-0,256 x+0,025 x^{2}-0,007 x^{3}$ & 0,173 & 0,657 & Estabilidade \\
\hline Gurupi & $Y=10,379+0,027 x-0,145 x^{2}+0,008 x^{3}$ & 0,279 & 0,429 & Estabilidade \\
\hline Dianópolis & $Y=3,429+0,251 x-0,080 x^{2}-0,002 x^{3}$ & 0,387 & 0,247 & Estabilidade \\
\hline
\end{tabular}

* Modelo: y = Indicadores epidemiológicos e operacionais da hanseníase para o Estado do Tocantins e coeficientes de detecção geral de hanseníase, coeficiente de detecção em menores de 15 anos e coeficiente de detecção de casos novos com grau 2 de incapacidade (por 100 mil habitantes) segundo regiões de saúde; $x=$ ano de ocorrência da doença - ano médio do período estudado (2006);

** R2: coeficiente de determinação.

\section{Discussão}

Neste estudo de âmbito estadual, os coeficientes de detecção geral da hanseníase apresentaram tendência decrescente no Estado do Tocantins, porém com padrões diferenciados entre as diversas regiões de saúde do estado. Houve persistência da transmissão ativa e diagnóstico tardio da doença, com padrões de hiperendemicidade em todo o território.
No período estudado, o registro de casos da doença na população geral foi maior no ano de 2006, e no grupo de menores de 15 anos foi em 2008. Esses registros foram impulsionados a partir de 2003 e permaneceram em crescimento até 2008, concomitantemente ao momento de municipalização das ações de controle da hanseníase no estado 12 . 
Figura 1

Detecção de casos novos de hanseníase (por 100 mil habitantes) na população geral e em menores de 15 anos de idade. Estado do Tocantins, Brasil, 2001-2012

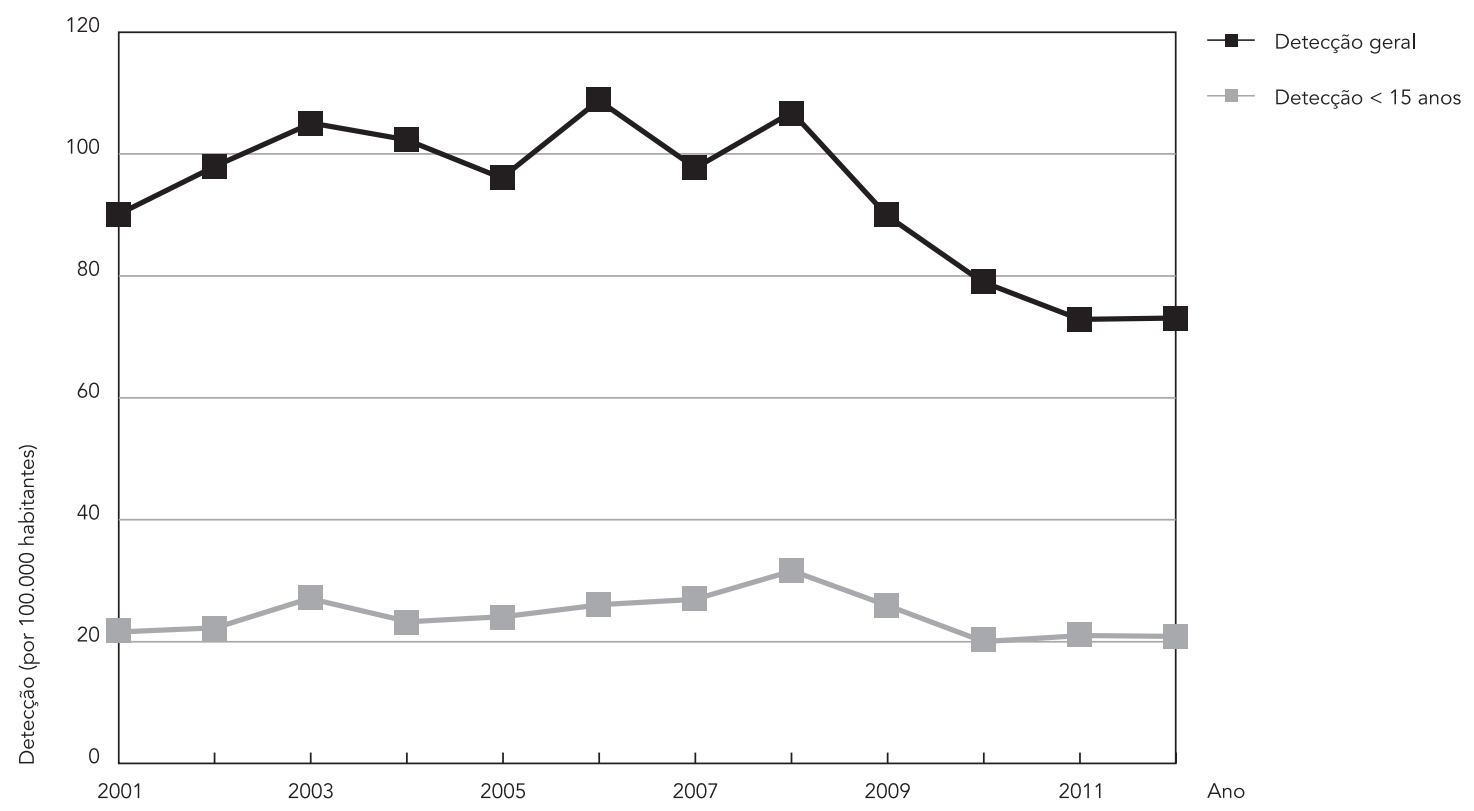

Figura 2

Proporção de casos detectados com graus 1 e 2 entre os avaliados, e detecção de casos com grau 2 (por 100 mil habitantes). Estado do Tocantins, Brasil, 2001-2012.

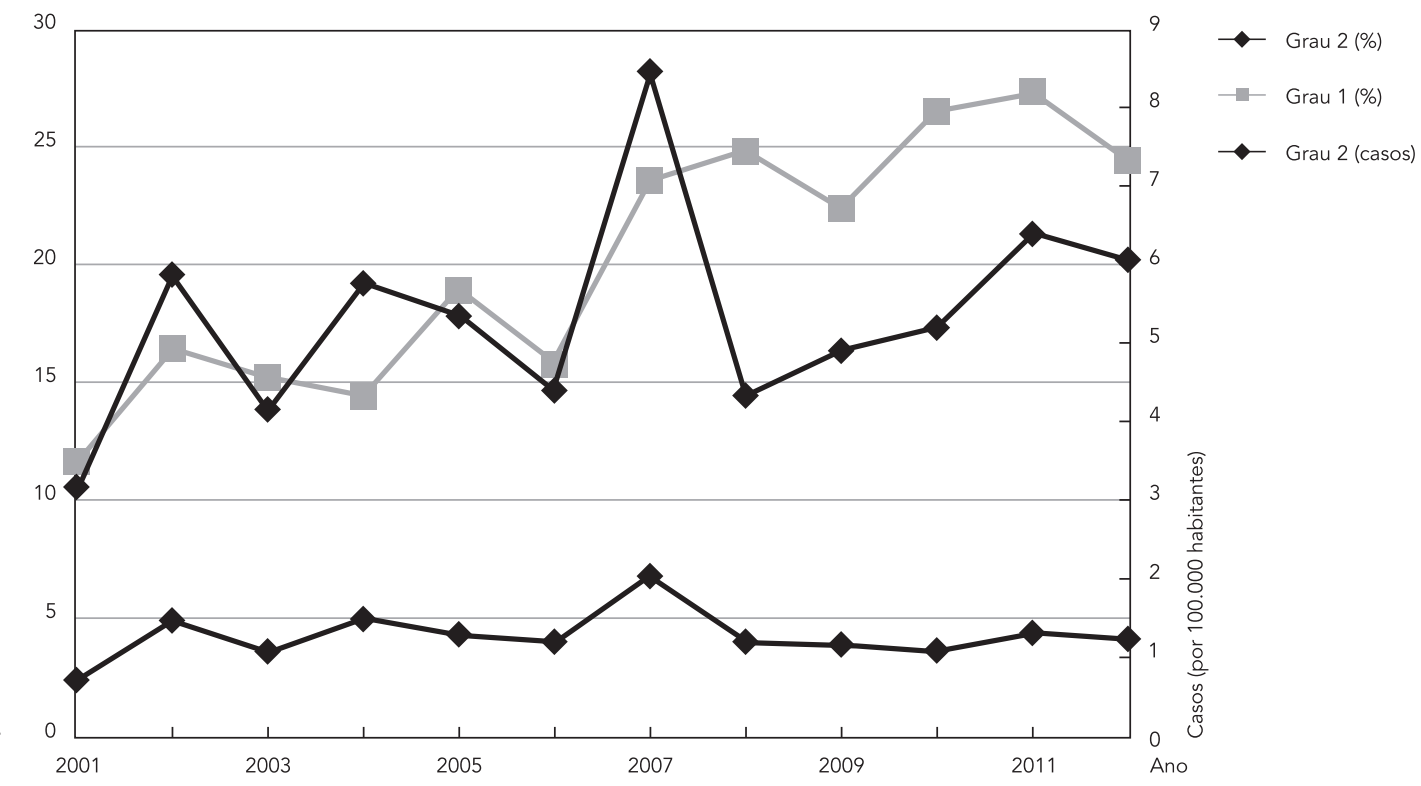


Proporção de casos de hanseníase detectados nas formas clínicas paucibacilares e multibacilares. Estado do Tocantins, Brasil, 2001-2012.

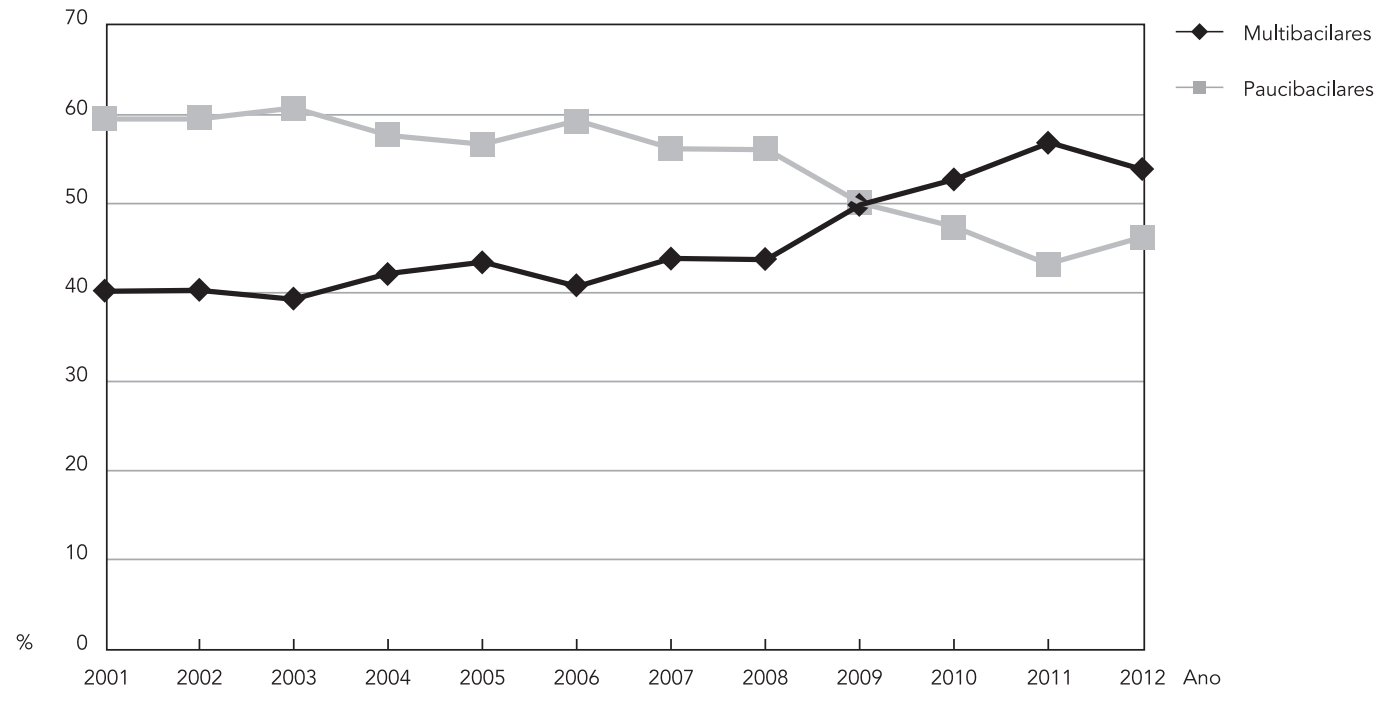

As regiões de saúde de Gurupi, Palmas e Araguaína apresentaram maior detecção no período, provavelmente por comporem as áreas de maior densidade populacional do estado (Instituto Brasileiro de Geografia e Estatística. http:// www.ibge.gov.br/estadosat/perfil.php?sigla=to, acessado em 12/Mar/2014), bem como pela disponibilidade de uma rede de serviços de referência e contrarreferência dando melhor suporte diagnóstico por profissionais especializados.

No contexto de diferentes cenários epidemiológicos da hanseníase, após mais de 30 anos da implementação da poliquimioterapia, ainda permanecem as discussões. As inovações estratégicas continuam na tentativa de melhorar a efetividade para atingir a meta de eliminação da hanseníase como problema de saúde pública nos países endêmicos 1,4,5.

A magnitude e a tendência da hanseníase descritas no período estudado apontam que, isoladamente, a municipalização das ações de controle, com total cobertura para o diagnóstico e tratamento da doença pela atenção primária, não é suficiente para o alcance da meta de eliminação.

A identificação de novos casos da doença frente às políticas públicas adotadas na Região Amazônica foi verificada por meio de modelos de regressão, e apontou que o Tocantins exerce influência positiva na produção de novos casos observados nesta região do Brasil 13.
Inversamente à tendência decrescente da detecção geral, a razão entre as classificações operacionais foi significativamente crescente para os casos multibacilares e para a proporção de casos com grau 1 de incapacidade física, apontando a persistência do diagnóstico tardio e a manutenção de fontes bacilíferas. A tendência da proporção de casos com grau 2 e do coeficiente de detecção de casos com grau 2 sinalizaram a constância da prevalência oculta e o atraso no diagnóstico pela estabilidade ao longo dos anos 14 .

Esses indicadores não acompanharam a tendência decrescente da detecção geral de casos novos e dificilmente se alcançará a redução do grau 2 de incapacidade em 35\%, conforme meta prevista pela OMS entre os anos de 2010 e 2015 15,16. O coeficiente de casos com grau 2 é um indicador que mensura a carga geral da doença na população atingida pela hanseníase no período de um ano, independentemente dos coeficientes de detecção de casos ou mesmo da prevalência 16 .

Diante desse cenário, o declínio do coeficiente de detecção geral da doença não pode ser atribuído à mudança no seu comportamento epidemiológico, pois a hanseníase não é uma doença infecciosa sensível às estratégias de eliminação, mas sim uma doença crônica, estável e de longo período de incubação com afinidade por desfavoráveis contextos sociais. Tampouco podem ser 
esperadas mudanças súbitas na sua ocorrência, como por exemplo, redução da incidência 4,6,17.

Uma possível hipótese para o decréscimo na detecção de casos novos no Tocantins é ter havido uma detecção intensificada, ocasionada por uma melhor cobertura dos programas de controle em virtude da municipalização dos serviços ${ }^{8}$. Isso, por sua vez, poderia ter contribuído para a queda da prevalência oculta nos últimos anos 1,6,14.

De fato, houve um incremento importante na proporção da cobertura populacional por equipes de saúde da família nos últimos 15 anos no Tocantins, que partiu de 4,3\% em janeiro de 1998 para $88,3 \%$ em dezembro de 2013 . A cobertura foi mantida acima de $60 \%$ e sustentável desde 2002 , com equipes de saúde da família em todos os 139 municípios 12,18.

Há ainda que se reconhecer o fortalecimento do Programa de Controle da Hanseníase no Tocantins, que se deu como consequência das diversas parcerias deste programa junto à sociedade civil e organizada, organizações não governamentais com aporte de pesquisas operacionais e assessoramento técnico 4,8,9,12, com o apoio da Leprosy Relief Association (LRA); parcerias com universidades por meio de projetos ensino/serviço; intensificação das ações de vigilância por intermédio da mídia (rádio, televisão e telefone); seminários; capacitação de profissionais da atenção primária à saúde no âmbito dos municípios; bem como campanhas municipais 12,19.

Outro aspecto relevante é a relação da hanseníase com a pobreza 20,21 . Nesse sentido, uma melhoria significante dos indicadores da doença pode ser reflexo de mais aporte às condições socioeconômicas para a população do estado, acompanhando a evolução do sistema de saúde estadual. Esse progresso fica patente no aumento da esperança de vida ao nascer de 60,3 anos em 1991 para 72,5 anos em 2010, na redução da mortalidade infantil de 36,5 por mil nascidos vivos em 2000 para 19,5 por mil nascidos vivos em 2010, e no declínio da extrema pobreza (medida pela proporção de pessoas com renda domiciliar per capita inferior a $\mathrm{R} \$ 70,00$ em agosto de 2010) de 31,8\% em 1991 para 10,2\% em 2010 19,22.

Essas hipóteses podem ser coerentes, pois após um aumento no número de casos diagnosticados, visualizou-se uma queda progressiva dessa ocorrência por meio dos modelos de regressão aplicados. Por outro lado, é preciso analisar esses dados de forma cautelosa, pois mudanças operacionais do programa de controle também influenciam na detecção de casos, como alterações na rotina de notificação de casos novos e saída do registro ativo por alta administrativa, após concluir o tempo previsto para tratamento no sistema de informação 4,6 .
Uma redução significativa na detecção de casos no Brasil e estados foi prevista a partir de 2010, com base na série histórica de 1980 a 2004 2,23. Contudo, uma queda brusca no coeficiente de detecção, se não for bem avaliada, pode vir a ser mal interpretada como uma evidência da capacidade de se controlar a hanseníase rapidamente no processo de eliminação ou após ele. Pois há que se reconhecer o risco de uma possível troca do declínio da prevalência conhecida pelo aumento da prevalência oculta 6 .

Quando projetada para o cenário mundial, a tendência de queda da hanseníase deve ser vista com prudência, pois as estratégias de eliminação contribuem lentamente para a redução da transmissão, sendo necessário o fortalecimento das ações de controle para além do ano de 2020, quando se espera observar uma redução importante na detecção de novos casos 24 .

No Brasil, foi observado o decréscimo na detecção de casos com grau 2 de incapacidade acompanhando a tendência de queda da detecção geral de casos novos. Porém, é importante destacar que a evolução dos indicadores de monitoramento da doença é heterogênea quando observada nos níveis global 16 , nacional, regional e local. Por isso é necessário fortalecer e intensificar a vigilância nos focos estatisticamente significantes de transmissão 25 .

No Estado do Tocantins, apesar da redução no registro de casos da doença, foram identificadas tendências desiguais na detecção geral por regiões de saúde. A estabilidade permanece nas regiões de Araguaína e Augustinópolis ao norte do estado e Dianópolis ao sudeste, e a detecção de casos em menores de 15 anos permanece estável para cinco das oito regiões. O comportamento epidemiológico da hanseníase observado em menores de 15 anos pode ser explicado pela persistência de focos ativos de transmissão, ou seja, aumento da cadeia de transmissão na população, como consequência de bacilos circulantes oriundos de casos multibacilares 2,26

A detecção de casos com grau 2 de incapacidade física permaneceu estável para todas as regiões do estado, mantendo o quadro epidemiológico de diagnóstico tardio. Esse cenário indica as dificuldades que o estado enfrenta para atingir a meta de redução do grau 2 de incapacidade proposta pela OMS 16. No Estado do Tocantins, essa meta é impraticável até 2015, pois essa proposta contribuirá apenas para redução da quantidade de pessoas vivendo com grau 2 no nível estadual, permanecendo as demandas represadas nas áreas de maior risco da doença 25 .

Esse padrão desigual da tendência dos indicadores da hanseníase pode ser reflexo de fatores como melhor cobertura das ações e serviços 
de saúde em alguns municípios e regiões, enquanto outros apresentam mais dificuldades de oferecer acesso e disponibilidade de serviços de saúde 12,18. Outros fatores podem ser as estreitas fronteiras geográficas, que facilitam a migração de pessoas de municípios de estados vizinhos considerados hiperendêmicos (Pará, Maranhão e Mato Grosso) para o Tocantins. Ressalte-se que importantes mudanças ocorreram no perfil epidemiológico da população no Estado do Tocantins, o mais novo do Brasil, o que propicia um crescimento demográfico marcante pelo consequente fluxo migratório 10,12. Em suma, os processos migratórios são potencializadores para a persistência da doença em diferentes regiões 27 .

Um dos componentes estratégicos do plano "Brasil Sem Miséria” é eliminar a hanseníase como problema de saúde pública até $2015^{3}$. Contudo, há mais de três décadas, a poliquimioterapia foi introduzida no tratamento da hanseníase e, ainda assim, não existem evidências claras do seu impacto na transmissão da doença 4,5. Apesar dos avanços nas ações de controle realizadas nos últimos 12 anos pelo Programa Estadual de Controle da Hanseníase no Estado de Tocantins, as medidas de controle precisam ser ajustadas para o alcance de indicadores mais baixos e próximos aos padrões esperados.

A interpretação dos resultados deve levar em consideração que este estudo pode apresentar limitações decorrentes da utilização de dados secundários, por possível inconsistência em relação à quantidade e à qualidade de suas informações. Além das limitações relacionadas ao preenchimento das notificações pelos profissionais de saúde, destacam-se fatores relacionados ao processamento dos dados que podem interferir na qualidade de dados no SINAN, como a inadequada digitação dos dados e as lacunas de preenchimento de variáveis provenientes dos serviços de saúde.
Para minimizar erros, lacunas e inconsistências, o banco de dados do SINAN nacional foi confrontado com os dados do banco do SINAN estadual, que foi disponibilizado pela Secretaria de Estado da Saúde do Tocantins por meio da área técnica responsável, fortalecendo a base de evidências deste estudo. O banco do SINAN estadual possui uma ampla cobertura e melhor qualidade das informações. Ressalte-se que a avaliação da completude do preenchimento das informações foi boa para as variáveis preenchidas no momento do diagnóstico e razoável para as variáveis de seguimento dos casos. Apesar das limitações mencionadas, os resultados mostram consistência interna e coerência com conhecimentos existentes sobre a hanseníase. São altamente representativos, já que incluíram todas as notificações de casos de residentes no estado, mesmo quando notificados em outras unidades federativas durante um período de mais de uma década.

Em conclusão, este estudo indica que a transmissão da hanseníase ainda é persistente no Estado do Tocantins, apesar da boa cobertura dos serviços de saúde nos últimos 12 anos. Os resultados apontam que, além das ações desenvolvidas pelo programa estadual de controle da doença, são necessárias novas abordagens de pesquisa para um melhor conhecimento sobre os mecanismos de transmissão de M. leprae 20,28, bem como reconhecer áreas de maior risco e suas potenciais fontes de infecção, e assim fornecer subsídios às ações de controle no estado. Esses achados reforçam ainda a necessidade de priorizar a atenção à hanseníase como condição crônica inserida efetivamente na rede de atenção do Sistema Único de Saúde (SUS). Além disso, recomendam-se a revisão e fortalecimento das ações de controle dos programas estadual e municipais para que estas sejam pautadas na realidade das diferentes regiões e contextos da doença no Estado do Tocantins. 


\section{Resumen}

La lepra es hiperendémica en el estado de Tocantins, Brasil. El objetivo del estudio fue analizar las tendencias de los indicadores de la lepra en Tocantins, 2001 2012. El análisis de los datos procedentes de las enfermedades de declaración obligatoria en el Sistema de Información sobre Dolencias Notificables (SINAN) incluía nuevos casos que viven en Tocantins. Se calcularon los indicadores de la lepra y se analizaron las tendencias temporales de regresión polinómica. Hubo una significativa tendencia a la baja, la detección general $\left(R^{2}=0,40 ; p<0,05\right)$ y el número de casos paucibacillar $\left(R^{2}=0,81\right)$. Fue una detección estable en $<15$ años $\left(R^{2}\right.$ $=0,48 ; p>0,05)$, la detección de los casos con discapacidad grado $2\left(R^{2}=0,37 ; p>0,05\right)$ y la proporción de casos con grado $2\left(R^{2}=0,49 ; p>0,05\right)$. Hubo un aumento significativo en la proporción de casos con discapacidad grado $1\left(R^{2}=0,82 ; p<0,05\right)$ y la proporción de casos multibacilares $\left(R^{2}=0,81 ; p<0,05\right)$. Tocantins cuenta con regiones de alta transmisión y un diagnóstico tardio de la lepra que apunta a la expansión heterogénea de la enfermedad en el análisis temporal.

Lepra; Enfermedades Desatendidas; Enfermedades Endémicas

\section{Colaboradores}

L. D. Monteiro, C. H. Alencar e J. Heukelbach participaram da concepção do projeto, coleta, análise e interpretação dos dados, redação do artigo e aprovação final da versão a ser publicada. F. R. Martins-Melo, A. L. Brito e M. S. Lima colaboraram na análise e interpretação dos dados, redação do artigo e aprovação final da versão a ser publicada.

\section{Agradecimentos}

Agradecemos o apoio do Conselho Nacional de Desenvolvimento Científico e Tecnológico; Departamento de Ciência e Tecnologia, Secretaria de Ciência, Tecnologia e Insumos Estratégicos, Ministério da Saúde; Coordenação de Aperfeiçoamento de Pessoal de Nível Superior; Secretaria de Estado da Saúde do Tocantins (SESAU-TO). À Luciana Ferreira Marques, Coordenadora de Doenças Transmissíveis e Não Transmissíveis da Superintendência de Vigilância e Proteção à Saúde da SESAU-TO pelo apoio, disponibilidade e concessão dos dados.

\section{Referências}

1. World Health Organization. Global leprosy situation, 2012. Wkly Epidemiol Rec 2012; 34:317-28.

2. Penna MLF, Oliveira ML, Penna GO. The epidemiological behaviour of leprosy in Brazil. Lepr Rev 2009; 80:332-44.

3. Ministério da Saúde. Brasil quer eliminar a hanseníase até 2015. http://www.brasil.gov.br/saude/ 2012/06/brasil-quer-eliminar-a-hanseniase-ate2015 (acessado em 13/Mar/2014).

4. Lockwood DN, Suneetha S. Leprosy: too complex a disease for a simple elimination paradigm. Bull World Health Organ 2005; 83:230-5.

5. Rodrigues LC, Lockwood DNJ. Leprosy now: epidemiology, progress, challenges, and research gaps. Lancet Infect Dis 2011; 11:464-70.
6. Penna ML, Penna GO. Trend of case detection and leprosy elimination in Brazil. Trop Med Int Health 2007; 12:647-50.

7. Heukelbach J, Chichava OA, Oliveira AR, Häfner $\mathrm{K}$, Walther F, Alencar CHM, et al. Interruption and defaulting of multidrug therapy against leprosy: population-based study in Brazil's savannah region. PLoS Negl Trop Dis 2011; 5:1031.

8. Monteiro LD, Alencar CHM, Barbosa JC, Braga KB, Castro MD, Heukelbach J. Incapacidades físicas em pessoas acometidas pela hanseníase no período pós-alta da poliquimioterapia em um município no Norte do Brasil. Cad Saúde Pública 2013; 29:909-20. 
9. Monteiro LD, Alencar CH, Barbosa JC, Novaes CCBS, Silva RCP, Heukelbach J. Limited activity and social participation after hospital discharge from leprosy treatment in a hyperendemic area in north Brazil. Rev Bras Epidemiol 2014; 17:91-104.

10. Ministério da Saúde. Portaria no 3.125, de 7 de outubro de 2010. Diário Oficial da União 2010; 15 out.

11. Latorre MRDO, Cardoso MRA. Análise de séries temporais em epidemiologia: uma introdução sobre os aspectos metodológicos. Rev Bras Epidemiol 2001; 4:145-52.

12. Coordenação de Doenças Transmissíveis e Não Transmissíveis, Secretaria de Estado da Saúde do Tocantins. Boletim Epidemiológico de Hanseníase 2013.

13. Silva FL, da Silva APL. Evolução de novos casos de Hanseníase frente às políticas públicas adotadas na Região Amazônica: um estudo através de modelos de regressão. Contribuciones a las Ciencias Sociales 2011; http://www.eumed.net/rev/ cccss $/ 15 /$.

14. Cunha MD, Cavaliere FAM, Hercules FM, Duraes SMB, Oliveira MLW, Matos HJ. Os indicadores da hanseníase e as estratégias de eliminação da doença, em município endêmico do Estado do Rio de Janeiro, Brasil. Cad Saúde Pública 2007; 23:1187-97.

15. World Health Organization. Enhanced global strategy for further reducing the disease burden due to leprosy (plan period: 2011-2015). New Delhi: World Health Organization; 2009.

16. Alberts CJ, Smith WCS, Meima A, Wang L, Richardus JH. Potential effect of the World Health Organization's 2011-2015 global leprosy strategy on the prevalence of grade 2 disability: a trend analysis. Bull World Health Organ 2011; 89:487-95.

17. Scheelbeek PF, Balagon MV, Orcullo FM, Maghanoy AA, Abellana J, Saunderson PR. A retrospective study of the epidemiology of leprosy in Cebu: an eleven-year profile. PLoS Negl Trop Dis 2013; 7:e 2444 .

18. Ministério da Saúde. Histórico de cobertura da saúde da família. http://dab.saude.gov.br/dab/his torico_cobertura_sf/historico_cobertura_sf_rela torio.php (acessado em 04/Mar/2014).
19. Penna MLF, Oliveira MLW, Carmo EH, Penna GO, Temporão JG. Influência do aumento do acesso à atenção básica no comportamento da taxa de detecção de hanseníase de 1980 a 2006. Rev Soc Bras Med Trop 2008; 41 Suppl 2:6-10.

20. Lockwood DN. Commentary: leprosy and poverty. Int J Epidemiol 2004; 33:269-70.

21. Kerr-Pontes LRS, Barreto ML, Evangelista CMN, Rodrigues LC, Heukelbach J, Feldmeier H. Socioeconomic, environmental, and behavioural risk factors for leprosy in North-east Brazil: results of a case-control study. Int J Epidemiol 2006; 35:9941000.

22. Programa das Nações Unidas para o Desenvolvimento. Atlas do Desenvolvimento Humano no Brasil 2013. http://atlasbrasil.org.br/2013/ (acessado em 12/Mar/2014).

23. Departamento de Vigilância Epidemiológica, Secretaria de Vigilância em Saúde, Ministério da Saúde. Hanseníase no Brasil: dados e indicadores selecionados. Brasília: Ministério da Saúde; 2009.

24. Meima A, Smith WCS, Van Oortmarssen GJ, Richardus JH, Habbema JDF. The future incidence of leprosy: a scenario analysis. Bull World Health Organ 2004; 82:373-80.

25. Oliveira ML, Grossi MA, Oliveira CF, Sena SA, Daxbacher E, Penna GO. Commitment to reducing disability: the Brazilian experience. Lepr Rev 2010; 81:342-5.

26. Alencar CH, Ramos Jr. AN, dos Santos ES, Richter J, Heukelbach J. Clusters of leprosy transmission and of late diagnosis in a highly endemic area in Brazil: focus on different spatial analysis approaches. Trop Med Int Health 2012; 17:518-25.

27. Alencar CHM, Ramos Jr. AN, Sena Neto SA, Murto C, Alencar MJF, Barbosa JC, et al. Diagnóstico da hanseníase fora do município de residência: uma abordagem espacial, 2001 a 2009. Cad Saúde Pública 2012; 28:1685-98.

28. Britton WJ, Lockwood DN. Leprosy. Lancet 2004; 363:1209-19.

Recebido em 19/Mai/2014

Versão final reapresentada em 21/Out/2014

Aprovado em 01/Dez/2014 